\title{
DIRICHLET'S PROBLEM FOR LINEAR ELLIPTIC PARTIAL DIFFERENTIAL EQUATIONS
}

\section{LARS GÅRDING}

Introduction. Consider a linear differential operator of order $v$,

$$
q=\sum_{|\beta| \leq \nu} q_{\beta}(x) D_{\beta}
$$

where the coefficients $q_{\beta}(x)$ are complex functions defined in some open subset $S$ of real $n$-dimensional space and $D_{\beta}$ derivatives $\left(\partial / \partial x_{1}\right)^{\beta_{1}} \ldots\left(\partial / \partial x_{n}\right)^{\beta_{n}}$ of orders $|\beta|=\beta_{1}+\ldots+\beta_{n}$ with respect to $x_{1}, \ldots, x_{n}$. For simplicity we shall assume that the coefficients are infinitely differentiable ${ }^{1}$. Restricting the summation on the right side of (1) to derivatives of order $v$ only, we get an operator $q_{v}$ which (if not zero) is called the principal part of $q$. Together with $q_{v}$ we consider the polynomial

$$
q_{\nu}(x, \xi)=\sum_{|\beta|=\nu} q_{\beta}(x) \xi_{\beta} \quad\left(\xi_{\beta}=\xi_{1}^{\beta_{1}} \ldots \xi_{n}^{\beta_{n}}\right)
$$

of the real variables $\xi_{1}, \ldots, \xi_{n}$. The operator $q$ is called elliptic in the region $S$ if $q_{\nu}(x, \xi) \neq 0$ when $x$ belongs to $S$ and $\xi \neq 0$. The simplest nontrivial example is the operator

$$
q=\frac{\partial}{\partial x_{1}}+i \frac{\partial}{\partial x_{2}} \quad(n=2) .
$$

Here $v=1, q=q_{v}$, and $q_{v}(x, \xi)=\xi_{1}+i \xi_{2} \neq 0$ if $\xi \neq 0$. The solutions $u=u(x)$ of the equation

$$
q u=0
$$

are all analytic functions of the variable $x_{1}+i x_{2}$. Other examples are Laplace's operator

and its powers.

$$
\frac{\partial^{2}}{\partial x_{1}{ }^{2}}+\ldots+\frac{\partial^{2}}{\partial x_{n}^{2}}
$$

Received April 1, 1953.

${ }^{1}$ An extension to sufficiently differentiable coefficients does not present any difficulties. 
We shall be concerned with weak solutions of the equation

$$
q u=h,
$$

where $h$ is an infinitely differentiable function in $S$. It is sufficient for our purpose to define a weak solution as a function $u$ which is integrable on all compact subsets of $S$ and satisfies the equation

$$
\int_{S} u(x) \overline{f \bar{q}(x)} d x=\int_{S} h(x) \overline{f(x)} d x
$$

whenever $f$ belongs to the set $H=H(S)$ of infinitely differentiable complex functions each of which vanishes outside some compact subset of $S$.

Here $\bar{q}$ is the formal adjoint of $q$ so that

$$
\bar{q} f(x)=\Sigma(-1)^{|\beta|} D_{\beta}\left(\overline{q_{\beta}(x)} f(x)\right) .
$$

Hence, if $u$ is $v$ times continuously differentiable we may integrate by parts in (3) and get $\int(q u(x)-h(x)) \overline{f(x)} d x=0$, so that $u$ is a solution of (2) in the ordinary sense. An important lemma by H. Weyl [18] asserts that if $q$ is Laplace's operator and $h=0$ then every weak solution of (2) after a correction on a null set becomes an ordinary solution, i. e., in this case, a harmonic function. This lemma is a special case of a theorem by Schwartz [15], p. 137, which states that every weak solution of (2) becomes infinitely differentiable after a correction on a nuli set, provided that every point of $S$ has a neighborhood in which a fundamental solution of $q$ with certain properties can be constructed. Recently F. John [10], [11] constructed such a fundamental solution assuming only that $q$ is elliptic with infinitely differentiable coefficients, and hence it follows that every weak solution of (2) becomes infinitely differentiable after a correction on a null set. If the fundamental solution is analytic, which is the case when the coefficients of $q$ are analytic, then every weak solution of (2) with $h=0$ is even analytic after a correction on a null set.

That Schwartz's theorem is in a certain sense characteristic for elliptic equations follows from a theorem by Petrowsky [14], which states that if $q$ has constant coefficients but is not elliptic, then the equation $q u=0$ has infinitely differentiable solutions which are not analytic.

It is the object of this paper to solve Dirichlet's problem for the equation (2) when (after multiplication of both sides by a suitable, non vanishing function of $x), q_{v}(x, \xi)$ is a positive definite polynomial ${ }^{2}, S$ is

\footnotetext{
${ }^{2}$ More generally: when the real part of $q_{v}$ is positive (see the end of section 3). This case has been treated by J. Leray, Sém. Bourbaki, May 1951, for equations with constant coefficients by a different method, and also by Vishik [17].
} 
bounded, and the coefficients of $q$ are sufficiently well-behaved. ${ }^{3}$ Since $q_{v}(x,-\xi)=(-1)^{v} q_{v}(x, \xi)$, the first assumption implies that $v$ has to be an even integer $2 m$. Roughly speaking, Dirichlet's problem is the following: To find a solution $u$ of (2) such that the derivatives of orders $<m$ of the function $u-g$, where $g$ is a given function in $S$, vanish at the boundary of $S$. Using a variant of the method of orthogonal projection by Zaremba [19] and Weyl [18], which may be considered as a form of Dirichlet's principle, we shall reduce this problem to a Fredholm equation. In the case $m=1$ we add nothing new. When $m>1$, the main difficulty arises from the fact that there are several possible Dirichlet integrals corresponding to the principal part of $q$ and that it may happen that none of them is positive definite. Nevertheless, any Dirichlet integral is bounded from below on the set of functions whose square integrals are 1 and which vanish in boundary strips of $S$ (Theorems 2.1 and 2.2). The proof of this fact is the essential point of the paper, which also contains a remark on Neumann's problem and ends with a short paragraph on Green's transformation and the vibration problem.-The main results were announced in [8] and partial results for constant coefficients in [5] and [6]. ${ }^{4}$

\section{The Hilbert space of functions with vanishing boundary values.} As in the introduction, define $H=H(S)$ as the set of all complex-valued infinitely differentiable functions vanishing outside compact subsets of an open subset $S$ of real $n$-space. Put

and

$$
f_{\beta}(x)=D_{\beta} f(x)
$$

$$
\left(f, f^{\prime}\right)_{j}=\int_{S^{\prime \beta \mid}=j} \sum_{\beta}(x) \overline{f^{\prime}(x)} d x .
$$

Closing $H$ with respect to the norm $|f|_{j}=(f, f)_{j}{ }^{1 / 2}$ we get a Hilbert space $\mathfrak{G}_{j}=\mathfrak{K}_{j}(S)$. If $j=0$ it consists of all (equivalence classes of) square integrable functions in $S$. We shall try to describe the spaces with $j>0$. Let us assume that $S$ is bounded. Then we have

\footnotetext{
${ }^{3}$ The exact conditions are found under a) and b) p. 60, c) p. 64, and d) p. 67 .

${ }^{4}$ Various circumstances have delayed the publication of this paper which was completed in December 1951. It overlaps with the papers [16] and [17] by Vishik, who in [17] also treats systems of equations. If in (1) we consider $q(x)$ as square matrices of order $l$, interpret ellipticity as $q_{v}(x, \xi)$ (more generally $\Re q_{v}(x, \xi)$ ) being positive definite for all $\xi$, and consider functions with values in $l$-dimensional unitary space, we get by formal changes the most important theorems of Vishik. However, the fundamental Theorem 2.1 is new. A review (with proofs) of the manuscript was given by L. Schwartz at the Bourbaki seminar in May 1952. A proof of Theorem 2.1 has also been found by F. Browder [2].
} 
Lemma 1.1. If $S$ is bounded there exist numbers $c_{k j}$ such that

$$
|f|_{k} \leqq c_{k j}|f|_{j}
$$

when $k \leqq j$ and $f$ belongs to $H$.

Proof. The inequality (1.2) is proved by repeated application of

$$
\int_{S}|f(x)|^{2} d x \leqq|S|^{2} \int_{S}\left|f_{k}(x)\right|^{2} d x \quad\left(f \in H, f_{k}=\partial f / \partial x_{k}\right),
$$

where $|S|$ is the diameter of $S$. This is Poincaré's inequality in the simplest case. To prove it we need only apply Schwarz's inequality to

$$
f(x)=\int_{-\infty}^{x_{k}} f_{k}\left(x_{1}, \ldots, t, \ldots, x_{n}\right) d t
$$

and afterwards integrate over $S$.

If $f^{v} \in H(v=1,2, \ldots)$ is a Cauchy sequence in $\mathfrak{H}_{m}$, it follows from (1.2) that it is also a Cauchy sequence in anyone of the spaces $\mathfrak{\mathscr { Y }}_{m-1}, \ldots, \mathfrak{S}_{0}$. Let $h$ be any infinitely differentiable function. Passing to the limit in

$$
\int f^{v}(x) h_{\beta}(x) d x=(-1)^{|\beta|} \int f^{v}{ }_{\beta}(x) h(x) d x,
$$

where $|\beta| \leqq m$, we get

$$
\int f(x) h_{\beta}(x) d x=(-1)^{|\beta|} \int f_{\beta}(x) h(x) d x,
$$

where $f$ and $f_{\beta}$ are certain square integrable functions vanishing outside $S$. It follows from (1.4) that $f_{\beta}$ is determined almost everywhere by $f$. We therefore take $f$ as a representative for the Cauchy sequence $f^{\nu}$. The same equation permits us to call $f_{\beta}$ a (generalized or weak) derivative ${ }^{5}$ of $f$. The fact that every $f \in \mathfrak{L}_{m}$ has square integrable derivatives of orders $\leqq m$ defined in the whole space, but vanishing outside $S$, means roughly speaking that $f$ and its derivatives of orders $<m$ vanish at the boundary of $S$. These functions belong to a class of functions studied by Nikodym [13] (see also Deny [4]). After a correction on a null set they are absolutely continuous on almost every straight line parallel to any of the axes, and after this correction they have vanishing limiting values almost everywhere on any smooth part of the boundary of $S$.

Lemma 1.1 has the following consequence:

Lemma 1.2. In $\mathfrak{S}_{m}$ the norms $|f|_{m}$ and $\|f\|_{m}=\left(|f|_{m}{ }^{2}+\ldots+|f|_{0}{ }^{2}\right)^{1 / 2}$ are equivalent.

5 The theory of such derivatives has been systematically developed by L. Schwartz [15]. 
Proof. It is clear that $|f|_{m} \leqq\|f\|_{m}$, and it follows from (1.2) that $\|f\|_{m}{ }^{2}=|f|_{m}{ }^{2}+\ldots+|f|_{0}{ }^{2} \leqq m c^{2}|f|_{m}{ }^{2}$ if $c$ is the largest of the numbers $c_{0 m}, \ldots, c_{m m}$.

It is sometimes useful to express the norms $|f|_{j}(f \in H)$ in terms of the Fourier transform

$$
F(\xi)=\int e^{i x \xi} f(x) d x \quad\left(x \xi=x_{1} \xi_{1}+\ldots+x_{n} \xi_{n}\right)
$$

of $f$. Then $f_{\beta}(x)$ has the Fourier transform $(-i)^{|\beta|} \xi_{\beta} F(\xi)$ and hence by Parseval's formula

$$
|f|_{j}^{2}=(2 \pi)^{-n} \int_{|\beta|=j} \xi_{\beta}{ }^{2}|F(\xi)|^{2} d \xi .
$$

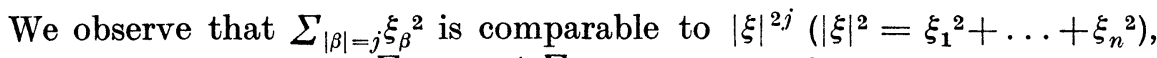
so that the quotient $\left(\sum_{|\beta|=j} \xi_{\beta}{ }^{2}\right) /\left(\sum_{|\xi|=m} \xi_{\beta}{ }^{2}+t\right)$ tends to zero as $t \rightarrow+\infty$, uniformly in $\xi$. This proves in particular that

$$
\lim _{t \rightarrow+\infty} \sup _{f \in H} \frac{|f|_{m-1}^{2}}{|f|_{m}^{2}+t|f|_{0}^{2}}=0 .
$$

The same formula leads to a simple proof of the following lemma.

Lemma 1.3. Let $D_{\alpha} f$ and $D_{\beta} f$ be two derivatives of $f$ of orders $\leqq m$, not both of order $m$, and let $a(x)$ be a bounded function in S. Then the equation

$$
\int a(x) D_{\alpha} f \overline{D_{\beta} f^{\prime}(x)} d x=\left(A f, f^{\prime}\right)_{m},
$$

where $f, f^{\prime}$ and Af belong to $\mathfrak{H}_{m}$, defines a completely continuous linear operator $A$.

Proof. By Schwarz's inequality we get that the left side of (1.7) is majorized by

$$
|a|\left|D_{\alpha} f\right|_{0}\left|D_{\beta} f^{\prime}\right|_{0},
$$

where $|a|=\sup |a(x)|$. Hence by Lemma 1.1 it is a continuous function of $f$ and $f^{\prime}$ in $\mathfrak{\mathfrak { E }}_{m}$, so that $A$ exists as a bounded linear operator. If for instance the order of $D_{\alpha}$ is less than $m$ we get

$$
\left|\left(A f, f^{\prime}\right)_{m}\right| \leqq C|f|_{m-1}\left|f^{\prime}\right|_{m},
$$

where $C$ is a constant, and hence

$$
|A f|_{m} \leqq C|f|_{m-1} \text {. }
$$

Now let $f^{v}(v=1,2 \ldots)$ be a sequence of elements in $H$ such that $\left|f^{\nu}\right|_{m} \leqq 1$. Then also the sequence $\left|f^{v}\right|_{0}$ is bounded and hence we may pick out a subsequence $f^{v^{\prime}}$ such that the Fourier transforms 


$$
F^{v^{\prime}}(\xi)=\int e^{i x \xi} f^{v^{\prime}}(x) d x
$$

which are uniformly bounded, converge for all $\xi$. Using the formulas (1.5) and (1.8) we get, $C_{1}$ and later $C_{2}$ denoting suitable constants,

$$
\begin{gathered}
\left|A f^{\nu^{\prime}}-A f^{\mu^{\prime}}\right|_{m^{2}} \leqq C^{2}\left|f^{\nu^{\prime}}-f^{\mu^{\prime}}\right|_{m-1}{ }^{2} \\
\leqq C_{1}{ }_{|\xi|<R}|\xi|^{2 m-2}\left|F^{\nu^{\prime}}(\xi)-F^{\mu^{\prime}}(\xi)\right|^{2} d \xi+C_{1}^{2} \int_{|\xi| \geqq R}|\xi|^{2 m-2}\left|F^{\nu^{\prime}}(\xi)-F^{\mu^{\prime}}(\xi)\right|^{2} d \xi .
\end{gathered}
$$

Here the first integral tends to zero when $\min \left(v^{\prime}, \mu^{\prime}\right) \rightarrow \infty$. The second integral does not exceed

$$
C_{1}{ }^{2} R^{-2} \int_{|\xi| \geqq R}|\xi|^{2 m}\left|F^{\nu^{\prime}}(\xi)-F^{\mu^{\prime}}(\xi)\right|^{2} d \xi \leqq C_{2}{ }^{2} R^{-2}\left|f^{\nu^{\prime}}-f^{\mu^{\prime}}\right|_{m}^{2} \leqq 4 C_{2}{ }^{2} R^{-2}
$$

and tends to zero with $1 / R$. Hence $\left|A f^{\nu^{\prime}}-A f^{\mu^{\prime}}\right|_{m} \rightarrow 0$ as $\min \left(\nu^{\prime}, \mu^{\prime}\right) \rightarrow \infty$, so that $A$ is completely continuous.

2. Dirichlet's integral. From now on let $m$ be a fixed positive integer. Suppose that for every $x$ in $S$ we are given a real polynomial $p(x, \xi)$ of the real variables $\xi_{1}, \ldots, \xi_{n}$ which is homogeneous of degree $2 m$.

We assume that

a) the coefficients of $p(x, \xi)$ are infinitely differentiable and uniformly continuous in $S$.

b) the polynomial is uniformly positive definite in $S$. More precisely: inf $p(x, \xi)$ has a positive lower bound in $S$. $|\xi|=1$

To such a polynomial we can always construct Dirichlet quadratic forms, i. e., expressions of the form

$$
\sum_{|\alpha|=|\beta|=m} p_{\alpha \beta}(x) f_{\alpha}(x) \overline{f_{\beta}(x)},
$$

where the coefficients $p_{\alpha \beta}(x)$ are real and symmetric, satisfy the condition a), and have the property that

$$
\sum_{|\alpha|=|\beta|=m} p_{\alpha \beta}(x) \xi_{\alpha} \xi_{\beta}=p(x, \xi) .
$$

To see that such forms do exist we write $p(x, \xi)$ in the form

$$
\sum q_{k_{1} \ldots k_{2 m}}(x) \xi_{k_{1}} \ldots \xi_{k_{2 m}}
$$

where the indices $k_{1}, \ldots, k_{2 m}$ range independently from 1 to $n$ and the coefficients $q_{k_{1} \ldots k_{2 m}}$ are symmetric. Then the expression

$$
\sum q_{i_{1} \ldots i_{m} k_{1} \ldots k_{m}}(x) f_{i_{1} \ldots i_{m}}(x) \overline{f_{k_{1} \ldots k_{m}}(x)} \quad\left(f_{i_{1} \ldots i_{m}}=\frac{\partial^{m} f(x)}{\partial x_{i_{1}} \ldots \partial x_{i_{m}}}\right)
$$


is a Dirichlet form. If $m=1$ there is only one Dirichlet form

$$
\sum q_{i k}(x) f_{i}(x) \overline{f_{k}(x)}
$$

and it is positive definite. If $m>1$, neither of these statements is true. Consider for instance the case $p(\xi)=\left(\xi_{1}{ }^{2}+\xi_{2}^{2}\right)^{2}$. Then

$$
\left|f_{11}(x)\right|^{2}+2(1-c)\left|f_{12}(x)\right|^{2}+\left|f_{22}(x)\right|^{2}+2 c \Re f_{11}(x) \overline{f_{22}(x)}
$$

is a Dirichlet form for any real $c$, and it is not positive definite ${ }^{6}$ if $|c| \geqq 1$.

Now pick out any Dirichlet form belonging to $p$ and put

$$
p\left(f, f^{\prime}\right)=\int_{S} \sum p_{\alpha \beta}(x) f_{\alpha}(x) \overline{f^{\prime}(x)} d x .
$$

If $f=f^{\prime}$ this expression is an analogue of Dirichlet's integral.

Omitting the index 0 in $\left(f, f^{\prime}\right)_{0}$, we shall write

and, later on, also

$$
\left(f, f^{\prime}\right)=\int_{S} f(x) \overline{f^{\prime}(x)} d x
$$

$$
p_{t}\left(f, f^{\prime}\right)=p\left(f, f^{\prime}\right)+t\left(f, f^{\prime}\right)
$$

where $t$ is a real number.

We can now formulate the principal result of the paper.

TheOREm 2.1. If $p(f, f)$ is any Dirichlet integral belonging to $p$ then ${ }^{7}$

$$
\inf _{f \in H} \frac{p(f, f)}{(f, f)}>-\infty .
$$

Proof. In the special case when $p(x, \xi)=p(\xi)$ is independent of $x$ it fol-

${ }^{6}$ It is interesting to note that if $p$ has constant coefficients then $p$ possesses a positive Dirichlet form,

$$
\sum p_{\alpha \beta} f_{\alpha} \bar{f}_{\beta} \geqq 0,
$$

if, and only if, $p(\xi)$ is a sum of squares. In fact, the Dirichlet form, if it is positive, can be written as a sum of squares,

and hence

$$
\sum_{\alpha}\left|\sum_{\beta} k_{\alpha \beta} f_{\beta}\right|^{2},
$$

$$
p(\xi)=\sum_{\alpha}\left|\sum_{\beta} k_{\alpha \beta} \xi_{\beta}\right|^{2}
$$

is itself a sum of squares. The converse is obvious. Now it is well known (Hilbert [9]) that there are positive definite polynomials which are not sums of squares, and such a polynomial has no positive Dirichlet form.

${ }^{7}$ It may happen that the lower bound is negative. In fact, let $1 \geqq h(x) \geqq \varepsilon>0$ in $S$. Then $p(x, \xi)=h^{2}(x)\left(\xi_{1}^{2}+\xi_{2}^{2}\right)^{2}$ has a Dirichlet integral

$$
p(f, f)=\int_{S} h^{2}\left(\left|f_{11}\right|^{2}+\left|f_{22}\right|^{2}+3 \Re f_{11} \overline{f_{22}}-\left|f_{12}\right|^{2}\right) d x .
$$

The integrand is negative if $f_{11}=f_{22}=0$ and $f_{12}=1$. Let $y=\left(y_{1}, y_{2}\right)$ be a point in $S$ 
lows directly from Parseval's formula that $p(f, f)$ is positive and that $(p(f, f))^{1 / 2}$ constitutes a norm in $\mathfrak{S}_{m}$ equivalent to $|f|_{m}$. In fact, let $f \in H$ and let

$$
F(\xi)=\int e^{i x \xi} f(x) d x
$$

be its Fourier transform. Then $f_{\alpha}(x)$ has the Fourier transform $(-i)^{m} \xi_{\alpha} F(\xi)$, and (2.3) together with (1.5) gives

and

$$
(f, f)_{m}=(2 \pi)^{-n} \int|F(\xi)|^{2}\left(\sum_{|\beta|=m} \xi_{\beta}^{2}\right) d \xi
$$

$$
p(f, f)=(2 \pi)^{-n} \int|F(\xi)|^{2} p(\xi) d \xi .
$$

Since the polynomial $p(\xi)$ is homogeneous of degree $2 m$ and positive definite, the quotient $\left(\sum \xi_{\beta}{ }^{2}\right)^{-1} p(\xi)$ is bounded from below by some positive number $c_{-}$and hence

$$
p(f, f) \geqq c_{-}|f|_{m}{ }^{2} \quad(f \in H) .
$$

This inequality can be used to take care of the general case. Let $w(\varrho)$ be the common module of continuity of all $p_{\alpha \beta}(x)$, i. e.,

$$
w(\varrho)=\sup _{\alpha, \beta, x, y}\left|p_{\alpha \beta}(x)-p_{\alpha \beta}(y)\right|,
$$

where $x$ and $y$ belong to $S$ and are subject to the restriction $|x-y|=$ $\left(\sum\left(x_{k}-y_{k}\right)^{2}\right)^{1 / 2} \leqq \varrho$. Let $T$ be a sphere of radius $\varrho$ and $\bar{x}$ its center. If $f$ belongs to $H(S \cap T)$ and $\bar{p}(f, f)$ is the Dirichlet integral corresponding to the polynomial $\bar{p}(\xi)=p(\bar{x}, \xi)$, we have

$$
\begin{aligned}
& |p(f, f)-\bar{p}(f, f)|=\left|\int_{\alpha, \beta}\left(p_{\alpha, \beta}(x)-p_{\alpha, \beta}(\bar{x})\right) \overline{f_{\alpha}(x)} f_{\beta}(x) d x\right| \\
& \quad \leqq \frac{1}{2} w(\varrho) \int \sum_{\alpha, \beta}\left(\left|f_{\alpha}(x)\right|^{2}+\left|f_{\beta}(x)\right|^{2}\right) d x=w(\varrho) d_{m}|f|_{m}{ }^{2},
\end{aligned}
$$

$d_{m}$ denoting the number of different derivatives of order $m$. Hence, by (2.5),

$$
p(f, f) \geqq \bar{p}(f, f)-|p(f, f)-\bar{p}(f, f)| \geqq\left(2 c-w(\varrho) d_{m}\right)|f|_{m}^{2}
$$

if $2 c=\inf _{y \in S} \inf _{|\xi|=1} p(y, \xi)$. By assumption $\mathrm{b}$ ), the number $c$ is positive, and by assumption a), the module of continuity $w(\varrho)$ tends to 0 with $\varrho$. Hence, if we choose $\varepsilon$ so small that $w(\varepsilon) d_{m} \leqq c$, it follows that

$$
p(f, f) \geqq c|f|_{m}^{2}
$$

and put $f(x)=\left(x_{1}-y_{1}\right)\left(x_{2}-y_{2}\right) b(x)$ where the function $b$ belongs to $H$ and equals 1 on a small circle with center $y$. Then $f$ is in $H$ and, if $h$ tends to the characteristic function of $C$, then $p(f, f)$ tends to a negative value. Hence $p(f, f)<0$ for some $f$ in $H$ if $h$ is chosen suitably. 
whenever $f$ belongs to $H(S \cap T)$ and $T$ is any sphere of radius $\varepsilon$. Now $S$ is bounded and hence we may cover $S$ by a finite set of spheres $T_{1}, \ldots, T_{N}$ of radius $\frac{1}{2} \varepsilon$. Let $2 T_{i}$ be the sphere concentric with $T_{i}$, but with radius $\varepsilon$. By a known device we may write

$$
1=\left|h_{1}^{2}(x)\right|+\ldots+\left|h_{N}^{2}(x)\right| \quad(x \in S),
$$

where the function $h_{i}(x)$ belongs to $H\left(2 T_{i}\right) \quad(i=1, \ldots, N)$. Let $f$ be in $H(S)$ and consider

$$
p(f, f)=\sum_{i} \int_{\alpha, \beta}\left|h_{i}{ }^{2}(x)\right| p_{\alpha \beta}(x) f_{\alpha}(x) \overline{f_{\beta}(x)} d x .
$$

Taking out one term $A_{i}$, we may write it as

$$
\left.\int \sum p_{\alpha \beta}(x)\left(h_{i}(x) f(x)\right)_{\alpha} \overline{\left(h_{i}(x) f(x)\right.}\right)_{\beta} d x-R_{i},
$$

where $R_{i}$ is a sum of integrals of products of a bounded function and two derivatives of $f$ of orders $\leqq m$, not both of orders $m$. Estimating $R_{i}$ in an obvious fashion and using Lemma 1.1, we get

$$
A_{i} \geqq p\left(h_{i} f, h_{i} f\right)-a_{i}|f|_{m}|f|_{m-1},
$$

where $a_{i}$ is a certain constant. But $h_{i} f$ belongs to $H\left(2 T_{i} \cap S\right)$ hence we may apply (2.6) with the result that

$$
A_{i} \geqq c\left|h_{i} f\right|_{m}^{2}-a_{i}|f|_{m}|f|_{m-1} \cdot
$$

By the same argument which led us to the estimate (2.7) of $A_{i}$ we conclude that

$$
\left(h_{i} f, h_{i} f\right)_{m} \geqq \int\left|h_{i}^{2}(x)\right| \sum_{|\alpha|=m}\left|f_{\alpha}(x)\right|^{2} d x-b_{i}|f|_{m}|f|_{m-1},
$$

where $b_{i}$ is a constant. Hence

$$
A_{i}=c \int\left|h_{i}{ }^{2}\right| \sum_{|\alpha|=m}\left|f_{\alpha}(x)\right|^{2} d x-\left(c b_{i}+a_{i}\right)|f|_{m}|f|_{m-1},
$$

so that, summing over $i$,

$$
p(f, f) \geqq c|f|_{m}{ }^{2}-a|f|_{m}|f|_{m-1},
$$

where $a=\Sigma\left(c b_{i}+a_{i}\right)$ and $f$ belongs to $H(S)$. This means that

$$
p_{t}(f, f)=p(f, f)+t(f, f) \geqq c|f|_{m}{ }^{2}+t|f|_{0}{ }^{2}-a|f|_{m}|f|_{m-1} .
$$

Let us put for a moment $|f|^{2}=c|f|_{m}{ }^{2}+t|f|_{0}{ }^{2}$. Then it follows that

$$
\begin{gathered}
p_{t}(f, f)= \\
p(f, f)+t(f, f) \geqq|f|^{2}\left(1-a|f|^{-2}|f|_{m}|f|_{m-1}\right) \geqq|f|^{2}\left(1-a c^{-1 / 2}|f|_{m-1}|f|^{-1}\right) .
\end{gathered}
$$


By virtue of formula (1.6), the quotient $|f|_{m-1} /|f|$ tends to zero as $t \rightarrow+\infty$, uniformly in $f$, and this shows that for $t$ large enough,

$$
p_{t}(f, f) \geqq \frac{1}{2}\left(c|f|_{m}^{2}+t|f|_{0}^{2}\right) .
$$

This proves our theorem and at the same time the following only apparently sharper result ${ }^{8}$ :

TheOREm 2.2. There exists a number $t_{0}$ such that, when $t>t_{0},\left(p_{t}(f, f)\right)^{1 / 2}$ constitutes a norm in $\mathfrak{G}_{m}$ equivalent to the norm $\left(|f|_{m}{ }^{2}+t|f|_{0}{ }^{2}\right)^{1 / 2}$. The equivalence is uniform for large $t$.

Proof. In fact, the last formula shows the uniform equivalence one way. The converse inequality

$$
p_{t}(f, f) \leqq c_{1}\left(|f|_{m}^{2}+t|f|_{0}^{2}\right),
$$

where $c_{1}$ is a constant which does not depend on $t$, follows trivially because

$$
\begin{gathered}
p(f, f)= \\
\int \sum p_{\alpha \beta}(x) f_{\alpha}(x) \overline{f_{\beta}(x)} d x \leqq \sup _{\alpha, \beta, x}\left|p_{\alpha \beta}(x)\right| \int \frac{1}{2} d_{m}\left(\sum_{\alpha}\left|f_{\alpha}(x)\right|^{2}+\sum_{\beta}\left|f_{\beta}(x)\right|^{2}\right) d x,
\end{gathered}
$$

where $d_{m}$, as above, denotes the number of different derivatives of order $m$.

3. Dirichlet's problem. Integrating by parts in $p_{t}\left(f, f^{\prime}\right)$ and assuming that $f$ and $f^{\prime}$ both belong to $H$, we get

$$
p_{t}\left(f, f^{\prime}\right)=\left(f, p_{t} f^{\prime}\right)=\left(p_{t} f, f^{\prime}\right),
$$

where $p_{t}$ is now a self-adjoint differential operator defined by

$$
p_{t} f(x)=(-1)^{m} \sum D_{\alpha}\left(p_{\alpha \beta}(x) D_{\beta} f(x)\right)+t f(x) .
$$

The most general linear differential operator $q$ with the same principal part as $p=p_{0}$ can be written as $q=p+r$, where

$$
r f(x)=\sum_{|\gamma|<2 m} r_{\gamma}(x) D_{\gamma} f(x)
$$

and $r_{\gamma}(x)$ are complex-valued functions. We assume that

c) the coefficients $r_{\gamma}(x)$ are infinitely differentiable in $S$ and their derivatives of orders $\leqq\left[\frac{1}{2}\left|\gamma^{\prime}\right|\right]$ are bounded in $S$, and put Dirichlet's problem in the following form:

\footnotetext{
${ }^{8}$ This follows if we apply Theorem 2.1 to the Dirichlet form $p(f, f)-\varepsilon(f, f)_{m}$ which corresponds to the polynomial $p(x, \xi)-\varepsilon \sum_{|\beta|=m} \xi_{\beta}^{2}$. If $\varepsilon>0$ is small enough, this polynomial has the properties a) and b).
} 
Given a function $g$ in $S$ such that $\|g\|_{m}=\left(|g|_{m}{ }^{2}+\ldots+|g|_{0}^{2}\right)^{1 / 2}<\infty$ and an infinitely differentiable function $h$ such that $|h|_{0}<\infty$. Find a solution $u$ of the diffential equation

$$
q u=h
$$

such that $g-u$ belongs to $\mathfrak{\mathfrak { B }}_{m}$, i. e., a solution $u$ having the same boundary values as $g$.

In order to solve this problem we use the well-known method of orthogonal projection by Zaremba [19] and Weyl [18]. Consider first the special case when $q=p_{t}$, where $t$ is a real number greater than the number $t_{0}$ appearing in Theorem 2.2. Then, if $f$ is in $H$, it follows from Lemma 1.1 and obvious estimates that the expression $p_{t}(g, f)-(h, f)$ is majorized by a constant times $|f|_{m}$ and hence it is an antilinear continuous function of $f$ in $\mathfrak{S}_{m}$. Hence, by Theorem 2.2 , there exists an element $g^{\prime}$ of $\mathfrak{H}_{m}$ such that ${ }^{9}$

$$
p_{t}(g, f)=p_{t}\left(g^{\prime}, f\right)+(h, f)
$$

for all $f$ in $H$. But $p_{t}(g, f)=\left(g, p_{t} f\right)$, and the equation

$$
p_{t}(k, f)=\left(k, p_{t} f\right),
$$

which is valid when $k$ and $f$ are in $H$, follows by a passage to the limit when $k=g^{\prime}$. Hence the square integrable function $u=g-g^{\prime}$ satisfies the equation

$$
\left(u, p_{t} f\right)=(h, f)
$$

when $f$ belongs to $H$. But then from Schwartz's theorem and John's construction of a fundamental solution it follows that $u$ is infinitely differentiable after a correction on a null set. Hence we may integrate by parts in (3.2) and get

$$
\left(p_{t} u-h, f\right)=0
$$

for all $f$ in $H$. Thus $p_{t} u=h$ and we have solved Dirichlet's problem in this special case.

Consider now the general case. With the differential operator $r$ we associate the bilinear form

$$
r\left(f, f^{\prime}\right)=\left(r f, f^{\prime}\right)=\left(f, \bar{r} f^{\prime}\right),
$$

${ }^{9}$ Among all $f$ in $\mathfrak{W}_{m}$ the function $g^{\prime}$ minimizes the expression

$$
p_{t}(g-f, g-f)-2 \Re(h, g-f),
$$

and hence our method is nothing but an application of Dirichlet's principle. The idea to consider directly the antilinear function $p_{t}(g, f)-(h, f)$ of $f$ seems to be due to Vishik [16] and has distinctive advantages when it comes to equations which are not self-adjoint. 
where $f$ and $f^{\prime}$ are in $H$ and $\bar{r}$ is the adjoint of $r$. After suitable integrations by parts we may write $r\left(f, f^{\prime}\right)$ as a sum of expressions of the form (1.7) where the function $a(x)$ is bounded by virtue of our assumption c). Hence $r\left(f, f^{\prime}\right)$ is a continuous function on $\mathfrak{S}_{m} \times \mathfrak{S}_{m}$.

If $u$ is a solution of our problem and $g^{\prime}=g-u$, then it follows from $q u=h$, by multiplication by a function $f$ in $H$ and integrations by parts, that

$$
q(g, f)=q\left(g^{\prime}, f\right)+(h, f),
$$

where $q(g, f)=p(g, f)+r(g, f)$, and analogously for $q\left(g^{\prime}, f\right)$. Conversely, if $g^{\prime}$ is an element of $\mathfrak{S}_{m}$ satisfying (3.3), then $u=g-g^{\prime}$ is a function in $\mathfrak{S}_{0}$ satisfying

$$
(u, \bar{q} f)=(h, f)
$$

for all $f$ in $H(\bar{q}=p+\bar{r}$ is the adjoint of $q)$. Hence, by Schwartz's theorem, $u$ is infinitely differentiable after a correction on a null set, and consequently it satisfies the equation $q u=h$. Thus we may concentrate upon (3.3).

Choose a number $t>t_{0}$ and put $r_{-t}=r-t$ so that $q=p_{t}+r_{-t}$. To begin with it is clear that $q(g, f)-(h, f)$ is a continuous antilinear function of $f$ in $\mathfrak{S}_{m}$, so that, by Theorem 2.2 , there exists an element $\tilde{g}$ in $\mathfrak{H}_{m}$ such that

$$
q(g, f)-(h, f)=p_{t}(\tilde{g}, f)
$$

for all $f$ in $H$. Moreover, according to Lemma 1.3 and Theorem 2.2, the equation

$$
r_{-t}\left(f, f^{\prime}\right)=p_{t}\left(R f, f^{\prime}\right),
$$

where $f, f^{\prime}$, and $R f$ are in $\mathfrak{H}_{m}$, defines a completely continuous linear operator $R$ on $\mathfrak{S}_{m}$ and hence (3.3) is equivalent to the equation

$$
\tilde{g}=g^{\prime}+R g^{\prime}
$$

to which the theorems of Fredholm are applicable. In particular, if the corresponding homogeneous equation $f+R f=0$ has only the solution $f=0$, then Dirichlet's problem has a unique solution for any $g$ and $h$ satisfying the given conditions.

Further, the two equations

$$
\begin{aligned}
& q u=f, \\
& \bar{q} v=h,
\end{aligned}
$$

where $u, v \in \mathfrak{H}_{m}$ and $f, h \in \mathfrak{H}_{0}$, constitute a Fredholm pair (Vishik [17] and Browder [1]). In fact, they may be written as

$$
q(u, k)=(f, k) \quad \text { for all } k \in H,
$$




$$
\bar{q}(v, k)=(h, k) \text { for all } k \in H,
$$

and hence, since $\bar{r}_{-t}\left(f, f^{\prime}\right)=\overline{r_{-t}\left(f^{\prime}, f\right)}=\overline{p_{t}\left(R f^{\prime}, f\right)}=p_{t}\left(f, R f^{\prime}\right)=p_{t}\left(R^{*} f, f^{\prime}\right)$ also as

$$
\begin{aligned}
& u+R u=C f, \\
& v+R^{*} v=C h,
\end{aligned}
$$

where $C$ is a completely continuous operator from $\mathfrak{\mathfrak { H }}_{0}$ to $\mathfrak{\mathfrak { H }}_{m}$, defined by $(f, k)=p_{t}(C f, k)$ for all $k \in H$. The two equations (3.6) obviously constitute a Fredholm pair.

Remark 1 . We may modify $q$ by putting $q=p+i p^{\prime}+r$ where $p^{\prime}$ is a differential operator of the same form (3.1) as $p=p_{0}$ and satisfies the condition

d) the coefficients of $p^{\prime}$ are real, infinitely differentiable, and bounded in $D$.

Then we may solve Dirichlet's problem also for the modified $q$. Because of $d$ ), the equation

$$
p^{\prime}(f, f)=p_{t}(A f, f),
$$

where $f$ and $A f$ are in $\mathfrak{S}_{m}$, defines a bounded self-adjoint linear operator $A$, and (3.4) becomes in this case

$$
\tilde{g}=g^{\prime}+i A g^{\prime}+R g^{\prime}
$$

to which the theorems of Fredholm are also applicable. The two equations (3.5) still constitute a Fredholm pair because the only change in (3.6) is that $R$ is replaced by $R+i A$ and $R^{*}$ by $R^{*}-i A$.

REMARK 2. The method of projection which we have used here also applies to other boundary value problems, e. g. Neumann's problem ${ }^{10}$. Then we have to replace $H$ by the set $\tilde{H}$ of all infinitely differentiable functions in $S$ with square integrable derivatives. The scalar products $\left(f, f^{\prime}\right)_{j}$ are defined as before. If $S$ has a smooth boundary, it is easy to see that the formula (1.2) is valid when we replace $|f|_{j}$ on the right side by $\left(|f|_{j}{ }^{2}+|f|_{0}^{2}\right)^{1 / 2}$. Lemma 1.2 has to be modified accordingly. The formula (1.5) is no longer true, but it is not difficult to see that (1.6) is still valid and also Lemma 1.3 if we replace the scalar product $\left(f, f^{\prime}\right)_{m}$ by $\left(f, f^{\prime}\right)_{m}+$ $\left(f, f^{\prime}\right)_{0}$.

Let us now assume that we have a polynomial $p(x, \xi)$ which has the property a) and that we have a corresponding Dirichlet form which is uniformly positive definite at all points of $S,{ }^{11}$

\footnotetext{
${ }^{10}$ Another method was given by Vishik [16].

${ }^{11}$ As we have seen, there are polynomials $p(x, \xi)$ for which such Dirichlet forms do not exist. In this connection it would be very interesting to know which polynomials have
} 


$$
\sum p_{\alpha \beta}(x) f_{\alpha}(x) \overline{f_{\beta}(x)} \geqq \varepsilon \sum\left|f_{\alpha}(x)\right|^{2} \quad(\varepsilon>0) .
$$

Then it is trivial that $p_{t}(f, f)$ and $|f|_{m}{ }^{2}+|f|_{0}{ }^{2}$ are equivalent square norms in $\tilde{H}$ if $t>0$. Let us denote the completed Hilbert space by $\tilde{\mathfrak{H}}_{m}$. If $g$ is a function defined in $S$ such that $p_{t} g$, as defined by (3.1), is square integrable, then $g$ has a projection $g^{\prime}$ in $\tilde{\mathfrak{H}}_{m}$ defined by the equation

$$
\left(p_{t} g, f\right)=p_{t}\left(g^{\prime}, f\right) \quad\left(f \in \tilde{\mathfrak{H}}_{m}\right) .
$$

Denoting by $f$ a function in $H$, we may integrate by parts and get

$$
\left(g-g^{\prime}, p_{t} f\right)=0 \quad(f \in H),
$$

which means that $u=g-g^{\prime}$ is infinitely differentiable and satisfies $p_{t} u=0$. Hence $p_{t} g^{\prime}$ exists and

$$
\left(p_{t} g^{\prime}, f\right)=p_{t}\left(g^{\prime}, f\right) \quad\left(f \in \tilde{\mathfrak{H}}_{m}\right) .
$$

If $g^{\prime}$ has derivatives of ordens $\leqq 2 m$ which are continuous in the closure of $S$, this equation implies that certain normal derivatives $\delta_{v} g^{\prime}$ of $g^{\prime}$ of order $\nu(m \leqq \nu<2 m)$ vanish at the boundary of $S$. If $m=1$ and $p$ is Laplace's operator, this means that the ordinary normal derivatives of $u$ and $g$ are equal, and hence we are justified in considering $u$ as the solution of the natural Neumann's problem for the equation $p_{t} u=0$, the boundary conditions being $\delta_{v}(u-g)=0$, which are implicit in the equation (3.7). The extension to differential operators with the same principal part as $p$ is immediate. It is also possible to treat by the same method a variety of boundary value problems analogous to Riemann's problem for Laplace's equation in the plane.

4. The vibration problem. For the differential operator

$$
q_{-\alpha}=p+r-\alpha,
$$

where $\alpha$ is a complex number, we may put the following vibration problem:

To find solutions in $\mathfrak{S}_{m}$ of the equation

$$
q_{-x} u=0 .
$$

Because of Schwartz's theorem this equation is equivalent to

$$
q_{-\alpha}(u, f)=0 \quad \text { for all } f \text { in } \mathfrak{S}_{m} .
$$

a Dirichlet form for which Theorem 2.1 is true when $f$ varies over $\tilde{H}$ and $S$ is a simple region, e.g. a square. It is conceivable that the Dirichlet form has to be positive in this ase. 
We are going to connect the solutions of (4.1) with Green's transformation $G_{t}$ defined in the next lemma.

LEMMA 4.1. If $t$ is sufficiently large, the equation

$$
(f, h)=q_{t}\left(G_{t} f, h\right),
$$

where $f, h$, and $G_{t} f$ are in $\mathfrak{S}_{m}$, defines a completely continuous linear operator $G_{t}$, whose inverse exists and is an extension of the differential operator $q_{t}$.

Proof. Choose a number $t>t_{0}$ (see Theorem 2.2). Then the equation $r\left(f, f^{\prime}\right)=p_{t}\left(R_{t} f, f^{\prime}\right)$, where $f, f^{\prime}$, and $R_{t} f$ are in $\mathfrak{H}_{m}$, defines a completely continuous linear operator for which, by virtue of Lemma 1.1 and Theorem 2.2, the square of the norm

is not greater than

$$
\left|R_{t}\right|_{t}^{2}=\sup \frac{\left|r\left(f, f^{\prime}\right)\right|^{2}}{p_{t}(f, f) p_{t}\left(f^{\prime}, f^{\prime}\right)}
$$

$$
c \sup \frac{\left|f^{\prime}\right|_{m}^{2}|f|_{m-1}^{2}}{\left(\left|f^{\prime}\right|_{m}^{2}+t\left|f^{\prime}\right|_{0}^{2}\right)\left(|f|_{m}^{2}+t|f|_{0}^{2}\right)} \leqq c \sup \frac{|f|_{m-1}^{2}}{|f|_{m}^{2}+t|f|_{0}^{2}},
$$

where $c$ is a constant independent of $t$. Hence $\left|R_{t}\right|_{t}$ tends to zero with $1 / t,(t>0)$. From Lemma 1.3 and Theorem 2.2 it follows that the equation $(f, h)=p_{t}\left(A_{t} f, h\right)$, where $f, h$, and $A_{t} f$ are in $\mathfrak{S}_{m}$, defines another completely continuous linear operator $A_{t}$. As $q_{t}\left(G_{t} f, h\right)=p_{t}\left(G_{t} f+R_{t} G_{t} f, h\right)$ we see that $(4.3)$ is equivalent to

$$
G_{t} f+R_{t} G_{t} f=A_{t} f
$$

Since $\left|R_{t}\right|_{t}$ tends to zero with $1 / t \quad(t>0)$,

$$
G_{t}=\left(1+R_{t}\right)^{-1} A_{t}
$$

exists for sufficiently large $t$ and is completely continuous. If $G_{t} f=0$ then $(f, h)=0$ for all $h$ in $H$ and hence $f=0$. Thus $G_{t}{ }^{-1}$ exists.

Consider the differential operator $q_{t}$ which we define as an operator on $\tilde{\mathfrak{S}}_{m}$ by letting its graph be the set of all pairs $\left\{f, q_{t} f\right\}$ where $f$ and $q_{t} f$ both belong to $\mathfrak{S}_{m}$ and $f$ is $2 m$ times continuously differentiable. Then $q_{t}\left(G_{t} q_{t} f, h\right)=\left(q_{t} f, h\right)=q_{t}(f, h)$ when $h$ is in $H$, so that $G_{t} q_{t} f=f$. This means that the graph of $q_{t}$ is contained in the set of all pairs $\left\{G_{t} f^{\prime}, f^{\prime}\right\}$ where $f^{\prime}$ lies in $\mathfrak{\mathfrak { Z }}_{m}$, i.e., in the graph of $G_{t}^{-1}$. This proves the lemma. We shall call $G_{t}$ Green's transformation.

Now suppose that $u \neq 0$ belongs to $\mathfrak{S}_{m}$ and satisfies (4.2). Then

$$
q_{t}(u, f)=(t+\alpha)(u, f)=(t+\alpha) q_{t}\left(G_{t} u, f\right)
$$

for all $f$ in $\mathfrak{\mathscr { B }}_{m}$. Hence $t+\alpha \neq 0$ and 


$$
G_{t} u=u /(t+\alpha) \text {. }
$$

Conversely, if $u \neq 0$ satisfies this equation, then

$$
(t+\alpha)(u, f)=(t+\alpha) q_{t}\left(G_{t} u, f\right)=q_{t}(u, f)
$$

for all $f$ in $\mathfrak{S}_{m}$ so that $u$ satisfies (4.2.). We have thus proved

Lemma 4.2. Any non-vanishing solution of (4.1) is an eigenfunction with the eigenvalue $1 /(t+\alpha)$ of every $G_{t}$ with $t$ large enough, and conversely.

In particular, if $u \neq 0$ satisfies (4.4) for one value of $t$, then the same equation follows for all values of $t$ for which $G_{t}$ makes sense. Since the eigenvalues of any $G_{t}$ form a sequence tending to 0 , it follows that the equation (4.1) has non-vanishing solutions only for a sequence of values $\alpha_{k}(k=1,2, \ldots)$ of $\alpha$ which tends to infinity.

REmark. If the degree of $q$ exceeds the number of variables, i. e., if $2 m>n$, we may apply the methods of Gårding [7] with only small modifications. The result is that for any $x$ in $S$ and $f$ in $\mathfrak{H}_{m}, \overline{f(x)}$ is an antilinear continuous function of $f$ and representable in the form

$$
\overline{f(x)}=q_{t}\left(G_{t}(x, \cdot), f\right),
$$

where $G_{t}(x, y)$ is the kernel (Green's function) of $G_{t}$,

$$
G_{t} f(x)=\int G_{t}(x, y) f(y) d y \text {. }
$$

For Green's function the asymptotic relation

$$
\lim _{t \rightarrow \infty} t^{1-\frac{n}{2 m}} G_{t}(x, y)=\delta_{x y}(2 \pi)^{-n} \int \frac{d \xi}{p(x, \xi)+1},
$$

where $\delta_{x y}=1$ when $x=y$ and $\delta_{x y}=0$ otherwise, may be proved as in Gårding [7]. If $q$ is also self-adjoint, i. e., if $q(f, f)$ is real for all $f$ in $H$, then $G_{t}$ is self-adjoint in the metric given by $q_{t}$ and has a complete set of mutually orthogonal eigenfunctions $\varphi_{1}, \varphi_{2}, \ldots$ We assume that they are labeled in such a way that the corresponding eigenvalues $1 /\left(t+\alpha_{1}\right)$, $1 /\left(t+\alpha_{2}\right), \ldots$ form a non-increasing sequence and normalize them so that $q_{t}\left(\varphi_{k}, \varphi_{k}\right)=\alpha_{k}+t$. Then

$$
\left(\varphi_{k}, \varphi_{j}\right)=q_{t}\left(G_{t} \varphi_{k}, \varphi_{j}\right)=\left(\alpha_{k}+t\right)^{-1} q_{t}\left(\varphi_{k}, \varphi_{j}\right)=\delta_{j k} .
$$

Green's function can then be represented as a uniformly convergent series

$$
G_{t}(x, y)=\sum_{1}^{\infty} \overline{\varphi_{k}(x)} \varphi_{k}(y)\left(\alpha_{k}+t\right)^{-1}
$$

Following Carleman [3], we may combine this formula with (4.5) and a 
Tauberian theorem by Hardy and Littlewood. The result is the following asymptotic formulas:

$$
\begin{gathered}
\lim N^{-1} \sum_{1}^{N} \overline{\varphi_{k}(x)} \varphi_{k}(y)=\delta_{x y} w_{p}(x) / w_{p}(S), \\
N(t)=\sum_{a_{k}<t} 1=(2 \pi)^{-n} w_{p}(S) t^{n / 2 m}(1+o(1)),
\end{gathered}
$$

where $w_{p}(x)=\int_{p(x, \xi)<1} d \xi, w_{p}(S)=\int_{S} w_{p}(x) d x$, and $o(1)$ tends to zero with $1 / t$. These formulas are well known in various special cases (see Gårding [7]) and are also valid without the restriction $2 m>n$, but then the proof is more complicated. ${ }^{12}$

12 It follows from a result announced by Keldych [12] that the eigenfunctions of $G_{t}$, together with certain associated functions, are complete in $\mathfrak{S}_{m}$ (in particular the number of eigenvalues is infinite) also when $q$ is not self-adjoint; and moreover (because this holds true for self-adjoint $q$ ) that the asymptotic formula (4.6) is valid also in general, if $2 m>n$ and $\alpha_{k}$ is replaced by $\Re \alpha_{k}$.

\section{BIBLIOGRAPHY}

1. F. Browder, The Dirichlet problem for linear elliptic equations of arbitrary even order with variable coefficients, Proc. Nat. Acad. Sci. U.S.A. 38 (1952), 230-235.

2. F. Browder, The Dirichlet and vibration problems for linear elliptic differential equations of arbitrary order, Proc. Nat. Acad. Sci. U.S.A. 38 (1952), 741-747.

3. T. Carleman, Propriétés asymptotiques des fonctions fondamentales des membranes vibrantes, Den åttonde skand. matematikerkongressen i Stockholm 1934, 34-44.

4. J. Deny, Les potentiels d'énergie finie, Acta Math. 82 (1950), 107-183.

5. L. Gårding, Le problème de Dirichlet pour les équations aux dérivées partielles elliptiques à coefficients constants, C. R. Acad. Sci. Paris 230 (1950), 1030-1032.

6. L. Gårding, Elliptic partial differential equations with constant coefficients. Proc. of the symposion on spectral theory and differential problems, Stillwater, Okl., 1951, 291-299.

7. L. Gårding, The asymptotic distribution of the eigenvalues and eigenfunctions of a general vibration problem, Kungl. fysiogr. sällsk. i Lund förh. 21 (1951), No 11.

8. L. Gårding, Le problème de Dirichlet pour les équations aux dérivées partielles elliptiques dans des domains bornés, C. R. Acad. Sci. Paris 233 (1951), 1554-1556.

9. D. Hilbert, Über die Darstellung definiter Formen als Summen von Formenquadraten, Math. Ann. 32 (1888), 342-350.

10. F. John, The fundamental solution of linear elliptic differential equations with analytic coefficients, Comm. Pure Appl. Math. 3 (1950), 273-304.

11. F. John, General properties of solutions of linear elliptic partial differential equations, Proc. of the symposion on spectral theory and differential problems, Stillwater, Okl., $1951,113-175$.

12. M. V. Keldych, On the eigenvalues and eigenfunctions of some classes of non-self-adjoint equations, Doklady Akad. Nauk SSSR 77 (1951), 11-14. (Russian.)

13. O. Nikodym, Sur une classe de fonctions considérées dans l'étude du problème de Dirichlet, Fund. Math. 21 (1933), 129-150. 
14. I. G. Petrowsky, Sur l'analyticité des solutions des systèmes d'équations différentielles, Rec. Math. Moscou, N. S. 5 (1937), 3-68.

15. L. Schwartz, Théorie des distributions I, Paris, 1950.

16. M. I. Vishik, The method of orthogonal and direct decomposition in the theory of elliptic partial differential equations, Mat. Sbornik 25 (1949), 189-234. (Russian.)

17. M. I. Vishik, On strongly elliptic systems of differential equations, Mat. Sbornik 29 (1951), 615-676. (Russian.)

18. H. Weyl, The method of orthogonal projection in potential theory, Duke Math. J. 7 (1940), 414-444.

19. S. Zaremba, Sur un problème toujours possible, comprenant à titre de cas particulier le problème de Dirichlet et celui de Neumann, J. Math. Pures Appl. (série 6) 9 (1927), $127-163$.

UNIVERSITY OF LUND, SWEDEN 